Draft Version July 11, 2018

Preprint typeset using $\mathrm{LAT}_{\mathrm{E} X} \mathrm{X}$ style emulateapj v. 6/22/04

\title{
HIGH ENERGY POLARIZATION OF BLAZARS: DETECTION PROSPECTS
}

\author{
N. Chakraborty ${ }^{1,2}$, V. Pavlidou ${ }^{3,4}$, B.D. Fields ${ }^{2}$ \\ Draft version July 11, 2018
}

\begin{abstract}
Emission from blazar jets in the ultraviolet, optical, and infrared is polarized. If these low energy photons were inverse-Compton scattered, the up-scattered high energy photons retain a fraction of the polarization. Current and future X-ray and gamma-ray polarimeters such as INTEGRAL-SPI, PoGOLITE, X-Calibur, GAP, GEMS-like missions, ASTRO-H, and POLARIX have the potential to discover polarized X-rays and gamma-rays from blazar jets for the first time. Detection of such polarization will open a qualitatively new window into high-energy blazar emission; actual measurements of polarization degree and angle will quantitatively test theories of jet emission mechanisms. We examine detection prospects of blazars by these polarimetry missions using examples of 3C 279, PKS 1510-089 and $3 \mathrm{C} 454.3$, bright sources with relatively high degrees of low energy polarization. We conclude that while balloon polarimeters will be challenged to detect blazars within reasonable observational times (with X-Calibur offering the most promising prospects), space-based missions should detect the brightest blazars for polarization fractions down to few percent. Typical flaring activity of blazars could boost the overall number of polarimetric detections by nearly a factor of 5-6 purely accounting for flux increase of the brightest of the comprehensive, all-sky, Fermi -LAT blazar distribution. Instantaneous increase in the number of detections is approximately a factor of 2 assuming duty cycle of $20 \%$ for every source. Detectability of particular blazars may be reduced if variations in flux and polarization fraction are anticorrelated. Simultaneous use of variability and polarization trends could guide the selection of blazars for high energy polarimetric observations.

Subject headings:
\end{abstract}

\section{INTRODUCTION}

Active Galactic Nuclei (AGNs) are some of the most luminous yet mysterious objects in the universe. Their particle and radiative emissions are powered by central supermassive black holes accreting matter. They are frequently observed to host relativistic jets, where bulk kinetic energy is converted to nonthermal random kinetic energy of electrons, radiation across the EM spectrum, and, possibly, particle emission (ions and neutrinos). Thus, these particles and radiation are messengers of the extreme astrophysical conditions in the core of active galaxies and their jets.

Blazars are AGNs where the observer's line of sight is closely aligned with the jet axis. Various properties of the radiation from blazars like the overall intensity, spectrum, and variability have been studied with multiwavelength observations. They have a non-thermal spectral energy distribution, with a low energy broadband peak in the range of radio to UV or even X-rays, and a second, high-energy peak, which starts from the X-ray band and can reach $\mathrm{TeV}$ or even higher energies. Their low energy peak is well explained by synchrotron from relativistic leptons, and as such can be highly polarized (e.g., Rybicki \& Lightman 1986). If higher energy emission is in part or in whole due to inverse Compton interactions of the polarized synchrotron component, then it too will

\footnotetext{
1 Max-Planck-Institut für Kernphysik, Saupfercheckweg 1, 69117 Heidelberg, Germany

2 Department of Astronomy and Department of Physics, University of Illinois, Urbana, IL

3 Department of Physics, University of Crete, 71003 Heraklion, Greece

4 Foundation for Research and Technology - Hellas, IESL, Voutes, 7110 Heraklion, Greece
}

retain some polarization.

While blazar polarization at low energies is wellestablished, blazar polarization at high energies has received much less attention, due to a lack of data. Indeed, there are few astrophysical sources of any kind that have measured high-energy polarization. Detections of polarized gamma-rays near the Crab Nebula and pulsar (e.g., Dean et al. 2008; Moran et al. 2013) are the most significant of the high energy polarimetric observations, with the Crab being used for calibrating polarization observations. Solar flares also have been observed in polarized X-rays (McConnell et al. 2003). Amongst transient sources, there are very few successful observations of gamma-ray bursts (GRBs) as listed in Chang et al. (2013). No high energy polarimetric data exists for blazars. This is in part due to the challenges in measurement of polarization in X-rays and soft gamma-rays. However, with numerous X-ray and soft gamma-ray polarimeters at various stages of planning, design and operation (e.g., Bellazzini et al. 2010; Pearce et al. 2012; Guo et al. 2013; Krawczynski et al. 2013; Costa et al. 2010; Yonetoku et al. 2011b; Mizuno 2012) and studies of optical / FIR polarization properties of blazars underway (e.g., Sasada et al. 2011; Ikejiri et al. 2011), a systematic study of high energy polarization from blazars is attracting renewed interest (Krawczynski 2012; Zhang \& Böttcher 2013; Krawczynski et al. 2013).

In this paper, we focus on detection prospects of X-ray and soft gamma-ray polarization of blazars with polarized seed photons in their jet. In Sec. 2 we discuss qualitatively different models for the high-energy emission from blazars and the conditions under which this emission is expected to be polarized. In Sec. 3, we discuss quantitatively the degree of polarization expected from 
inverse-Compton scattering of polarized low energy photons by relativistic electrons in the jet with a power-law distribution. We estimate polarization values for three of the brightest blazars with fairly high degrees of polarization in the infra-red as an illustration. The minimum detectable polarization (MDP) for various telescopes in general is reviewed in Sec. 4. Sec. 5 lists the chosen brightest blazars and the observing time required to detect the polarization for different polarimeters from the MDP formula. In section [6 we discuss the influence of flaring on detection prospects guided by observational trends of polarization and flaring. We discuss our conclusions along with future work in section 7 .

\section{HIGH-ENERGY POLARIZATION IN BLAZARS: QUALITATIVE DISCUSSION}

Blazar broadband spectral energy distributions exhibit two broad peaks. The first, lower energy peak is usually attributed to synchrotron emission from leptons that provides a good explanation of the spectrum. This suggests that peak emission is intrinsically polarized (Rvbicki \& Lightman 1986). It ranges from radio to optical and UV, and, for some high-synchrotronpeaked blazars, to as high an energy as X-rays. Observations of flat spectrum radio quasars (FSRQs) and low energy peaked BL LAC (LBL) objects at low energies (radio to optical) do indeed confirm that the low-energy peak emission is polarized(e.g., Lazzati 2006; Rossi et al. 2004; Wardle \& Kronberg 1974; Agudo et al. 2010; Fujiwara et al. 2012; ?).The high energy peaked BL LACs on the other hand have their first peaks in the Xray regime. X-ray polarimetry can help confirm the synchrotron origin of this peak. In this paper, we restrict ourselves to the blazars where polarimetric observations exist at low energies, i.e. to the FSRQs and LBLs.

The second high-energy peak in blazar spectra is less well understood and a matter of active debate. It is typically associated to inverse-Compton scattering of low energy photons. The emission can be purely leptonic, in which case primary accelerated electrons are the ones responsible for upscattering the lower-energy photons. Or it can involve hadronic processes as well. In the latter case, the primary accelerated particles also include protons and ions, and part of the high-energy emission is produced as a direct product of hadronic interactions (decay of neutral pions into gamma rays and proton synchrotron) and part through inverse Compton interactions of relativistic particles produced in hadronicallyinduced electromagnetic cascades. For the former case of inverse-Compton emission by primary electrons, seed photons can either originate in external sources (External Compton mechanism, EC) [e.g. Mever et al. (2012) and earlier references therein] such as the accretion disk and the broad-line region or from internal sources within the jet like the synchrotron photons [e.g. Zacharias \& Schlickeiser (2012) and earlier references therein] (self-synchrotron Compton, SSC).

In the SSC case, the high-energy emission retains a fraction of the synchrotron photon polarization due to the nature of the inverse Compton scattering process (Bonometto et al. 1970; McNamara et al. 2009: Krawczynski 2012; Zhang \& Böttcher 2013). The EC mechanism, on the other hand, is generally expected to produce lower degree of polarization from both analyti- cal calculations (Bonometto et al. 1970) and simulations (Krawczynski 2012). Simulations in McNamara et al. (2009) find higher degrees of polarization for both EC and SSC.

Polarization is a key ingredient in the multimessenger understanding of blazars. It can distinguish between different emission mechanisms of blazars such as hadronic and leptonic and even between different leptonic models as mentioned in, e.g., McNamara et al. (2009); Krawczynski (2012); Krawczynski et al. (2013) and demonstrated in a model-dependent way in Zhang \& Böttcher (2013). Blazar variability can serve as an additional diagnostic for monitoring the relation between the jet activity and polarization. This will also allow measurement of polarization during the flaring states of those blazars whose polarization would, under normal circumstances, be undetectable. We examine the effect of flux increase due to flaring of blazars in general. The detailed study of relations between variability and polarization for individual blazars is left for future work.

\section{DEGREE OF HIGH ENERGY POLARIZATION OF} BLAZARS: EXPECTATIONS

\subsection{High energy polarization from inverse-Compton scattering of low energy photons}

Polarization is quantified in terms of the degree of polarization, $\Pi$ which is the fraction of polarized light, and by the polarization position angle, $\psi$. Bonometto et al. (1970) calculated the polarization of photons produced by inverse-Compton scattering of a monochromatic beam of photons by an unpolarized electron distribution; both populations are assumed to have no spatio-temporal variations. In this calculation, the initial and scattered photon energies ( $\epsilon$ and $E_{\gamma}$ respectively) satisfy $E_{\gamma} \gg \epsilon$ and $E_{\gamma} \epsilon / m_{\mathrm{e}}^{2} \ll 1$ (Thomspon limit) where $m_{\mathrm{e}}$ is electron mass in energy units ; both of these should be valid for blazar emission up to $\sim 100 \mathrm{GeV}$.

Krawczynski (2012) revisited this calculation, both analytically and numerically, and confirmed the results of Bonometto et al. (1970). Krawczynski (2012) also computed, for the first time, the polarization from inverseCompton scattering by an isotropic distribution of electrons in the Klein-Nishina regime. For a photon spectrum generated by an isotropic distribution of electrons with a power-law energy spectrum of index $p$ upscattering mono-energetic, unidirectional, $\Pi_{\text {init }}=100 \%$ polarized photons, the resulting polarization fraction is (Krawczynski 2012)

$$
\Pi_{e, \text { powerlaw }}=\frac{(1+p)(3+p)}{\left(11+4 p+p^{2}\right)} .
$$

The retained polarization is substantial: for $p=2(3)$, $\Pi_{e, \text { powerlaw }} \approx 65 \%$ (75\%). These numbers assume the polarization and propagation directions of the polarized seed photons are perfectly aligned. In general, as shown in Krawczynski (2012), this retained fraction depends on the polarization angle, the angle between the seed photon and the magnetic field direction, the spectral index, etc. Evaluating this complex geometric factor for each blazar is beyond the scope of this work. But in order to account for this effect, we make the simplifying assumption that the polarization and propagation direction of the seed photons are at a fixed average angle to retain a fraction, 
$\eta_{\text {avg }}=80 \%$ of polarization. This is a reasonable number for blazar electron indices $\approx 2$ according to Krawczynski (2012). For an arbitrary initial polarization degree $\Pi_{i n i t}$, the upscattered polarization fraction becomes

$$
\Pi_{\text {upscatter }}=\eta_{\text {avg }} \Pi_{e, \text { powerlaw }} \Pi_{\text {init }}
$$

\subsection{Motivation for using observed polarization of low energy seed photons}

For blazars, the initial ("seed") polarization $\Pi_{\text {init }}$ is that of the low-energy photons, which themselves arise from synchrotron emission. Theoretically, for a powerlaw distribution of electrons, the degree of polarization for synchrotron is (e.g., Rybicki \& Lightman 1986),

$$
\Pi_{\text {sync }}=\frac{p+1}{p+7 / 3} \text {. }
$$

This would give large values, $\Pi_{\text {init }} \approx 70 \%$ for typical blazars where $p=2-3$. Optical observations show a high degree of polarization (e.g., Angel \& Stockman 1980) always lower than this maximum synchrotron limit in several cases. This is because, as various effects like Faraday rotation and inhomogeneities in the magnetic field as also presence of thermal components can reduce this value (Netzer 2013). Fermi-detected blazars with low degrees of polarization in the optical and IR have been observed (Fujiwara et al. 2012; ?). In light of the diversity of the observed low-energy blazar polarization, we will restrict our predictions only to blazars with observed values of $\Pi_{\text {init }}$. We thus propose to test empirically the relation between degrees of low energy polarization and high energy polarization.

In general, $\Pi$ is a function of the direction of target photons and energy of the scattered photons. Figures 8 and 9 in Krawczynski (2012) show $\Pi$ to be an increasing function of the scattered photon energy, from energies of $\sim 100 \mathrm{keV}$ to nearly $100 \mathrm{MeV}$ 's or so. This suggests that soft gamma-rays are likely to be polarized. In general, this depends upon the target photons for the particular blazar type. However, a detailed calculation appropriate at hard X-ray / soft gamma-ray energies is needed to show this explicitly. This is not the focus of this paper.

\section{DETECTION SENSITIVITY OF TELESCOPES: MINIMUM DETECTABLE POLARIZATION}

The detectability of polarization in a given source of certain strength by a given detector is quantified in terms of the minimum degree of polarization the detector can establish (the minimum detectable polarization, MDP). The MDP is the minimum fraction polarized intensity detectable given the strength of the source, $R_{S}$ relative to the background, $R_{\mathrm{bg}}$ in a given exposure time, T. The MDP of a source producing a partially polarized signal, $R_{\mathrm{S}}$ counts $\mathrm{sec}^{-1}$, is given by (Novick 1975; Weisskopf et al. 2010a; Kalemci et al. 2004),

$$
M D P=\frac{n_{\sigma}}{\mu R_{S}} \sqrt{\frac{2\left(R_{S}+R_{\mathrm{bg}}\right)}{T}},
$$

$n_{\sigma}$ is the detection significance, $R_{\mathrm{bg}}$ is the background count rate, and $\mu$ is the modulation factor, which quantifies the detector response to polarized light (with $\mu=1$ being perfect polarization sensitivity and $\mu=0$ being no polarization sensitivity). Given a detector with a certain modulation factor $\mu$ and background $R_{\mathrm{bg}}$ counts $\mathrm{sec}^{-1}$,
Eq. (4) can be inverted to determine the amount of time required to detect a source producing $R_{S}$ counts $\mathrm{sec}^{-1}$.

The observation time required to detect that a certain signal is polarized at a certain degree is different from the time required to make a measurement (both polarization degree and angle) of its polarization with some given uncertainty. A measurement of the source's polarization properties constitutes the joint measurement of both the amplitude (degree, $\Pi$ ) and phase (related to the position angle, $\psi$ ) of polarization as opposed to simply the amplitude. This difference is discussed by Weisskopf et al. (2010a); Strohmayer \& Kallman (2013) and the heart of the issue is that more photons are needed in order to jointly measure both the degree and position angle, than to simply establish that the source is polarized at a certain degree. Following the analysis in both Weisskopf et al. (2010a); Strohmayer \& Kallman (2013) and their suggested prescription, in this paper we make this distinction between the time required for a 3-sigma measurement of the MDP degree, $\Pi$ alone (establish polarization degree with only $1 \%$ probability for the deviation from zero-polarization to be caused by chance) and the time required for a joint 3-sigma measurement of the degree, $\Pi$, corresponding to the MDP and position angle, $\psi$. We tabulate both these times for the selected sources in section 5 . The times differ by a factor $\approx n_{\sigma}^{2} / 4=2.25$ for the polarization degrees of $\Pi \approx 10 \%$.

The GEMS White Paper (Krawczynski et al. 2013) proposes for GEMS-like missions a strategy similar to what we describe below. They emphasise using archival data on low energy polarization in order to motivate observations of high energy polarization. In this work we extend such calculations to polarimeters beyond GEMSlike missions, and explicitly consider the effects of flux increase during blazar flaring. Based on this, we motivate the possibility of simultaneous polarization observations of blazars at low and high energies taking advantage of current and future low-energy blazar polarization monitoring programs, such as the RoboPol optopolarimetric program (?), and the F-GAMMA(Angelakis et al. 2010), and OVRO (Richards et al. 2011) programs in radio frequencies.

\section{DETECTION PROSPECTS OF BLAZARS}

\subsection{Selection of test cases}

We use blazars in Ikejiri et al. (2011) and Fujiwara et al. (2012) (selected from the Fermi LAT catalog and studied in IR polarization) as the pool from which to select a few good candidates for high-energy polarization detection and use them as demonstration cases in our calculations below. Some of these are very bright, some possess a high degree of polarization, while others are variable and are good candidates for temporal studies. Thus, there are several alternate ways of selecting candidate blazars for polarimetric studies depending on both blazar characteristics and observability. 3C 279, PKS 1510-089 and 3C 454.3 are 3 of the brightest blazars that have the highest measured IR polarization recorded by various polarimeters as listed in Ikejiri et al. (2011); Fujiwara et al. (2012). In addition, they have shown significant variability (Abdo et al. 2010a; Sasada et al. 2011; Wehrle et al. 2012; Jorstad et al. 2010). Their flaring behaviour make them promising candidates a priori for polarization 
measurements as we will comment on in section [6. For these reasons, we will focus on these three sources for the remainder of this section.

\subsection{High-energy polarimeters}

Both main instruments on board INTEGRAL, IBIS and SPI, have reported polarization measurements of GRBs (Götz et al. 2013, 2009; McGlvnn et al. 2007, etc.,). Thus, it is natural to check prospects of blazar measurements with INTEGRAL. In addition, there are a number of polarimeters in various stages of planning, commissioning and operation. We explore GEMS-like missions (Krawczynski et al. 2013) as well as other instruments (Bellazzini et al. 2010) : balloon based polarimeters PoGOLITE (Pearce et al. 2012) and X-Calibur (Guo et al. 2013); space based instruments ASTRO-H (Mizuno 2012) and POLARIX (Costa et al. 2010), which are in the stage of planning; and the Gamma-Ray Burst Polarimeter (GAP) (Yonetoku et al. 2011b), which is already functional and has successfully detected polarized signals from some GRBs.

GAP on board IKAROS is designed specifically for GRBs and was able to detect polarized signals from GRBs 110301A, 110721A and 100826A (Yonetoku et al. 2012, 2011a). Despite being suited for prompt polarimetry of GRBs, it is worth looking at prospects of blazar polarimetry with GAP, particularly those which have demonstrated high flux variations like the 3 we consider.

\subsection{Strategy of calculations}

We use observed polarization values, $\Pi_{\text {init }}$ to determine the degree of high energy polarization of a blazar,

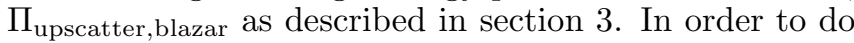
this, we scale from the sensitivity of polarimeters to a Crab-like source for each blazar. We denote this source using the subscript "calib" (for calibrator), as this is essentially a way of calibrating the response of the polarimeter. The sensitivity of polarimeters are given in terms of the amount of time taken to detect the MDP from a source of a given flux (typically expressed in mCrab units) in accordance with Eq. 4.

Scaling from the Crab using Eq. (4), we get then the required observation times, $T_{\text {obs }}$. Here there are 3 cases, if the background for the polarization signal is same for both the calibrator and blazar, noting that this may not always hold true in practice. First, when the source signal is much higher than the background for both the calibrator source and the blazar i.e. $R_{\text {blazar }}, R_{\text {calib }} \gg$ $R_{\text {bg. }}$. Second, when the background dominates over the signal for both i.e., $R_{\text {blazar }}, R_{\text {calib }} \ll R_{\text {bg }}$. Third, when the calibrator signal dominates the background, but the background dominates the blazar signal i.e., $R_{\text {calib }} \gg R_{\text {bg }} \gg R_{\text {blazar. }}$ A fourth case would occur if the blazar signal dominates the background, but the calibrator doesn't. However, it is highly unlikely that the blazars are brighter than the calibrator source which is typically the Crab.

In the first case, when the blazar and calibrator count rates are much higher than the background i.e. $R_{\text {calib }}, R_{\text {blazar }} \gg R_{\text {bg }}$, the required observation times are given by,

$$
\left.\frac{T_{\text {obs,blazar }}}{T_{\text {obs }, \text { calib }}}\right|_{\text {sig dom }}=\left(\frac{R_{\text {calib }}}{R_{\text {blazar }}}\right)\left(\frac{M D P_{\text {calib }}}{\Pi_{\text {upscatter,blazar }}}\right)^{2}
$$

In the second case, when both the blazar and calibrator count rates are much lower than the background, i.e., $R_{\text {calib }}, R_{\text {blazar }} \ll R_{\text {bg }}$, the required observation times are given by,

$$
\left.\frac{T_{\text {obs,blazar }}}{T_{\text {obs,calib }}}\right|_{\text {bgd dom }}=\left(\frac{R_{\text {calib }}}{R_{\text {blazar }}}\right)^{2}\left(\frac{M D P_{\text {calib }}}{\Pi_{\text {upscatter,blazar }}}\right)^{2}(6)
$$

respectively. This is true provided the background is the same for the calibrator and the blazar such that it drops out from the ratio of observing times. In the third case i.e., $R_{\text {calib }} \gg R_{\mathrm{bg}} \gg R_{\text {blazar }}$, the observation time is given by,

$$
\frac{\left.T_{\text {obs,blazar }}\right|_{\text {bgd dom }}}{\left.T_{\text {obs,calib }}\right|_{\text {sig dom }}}=\left(\frac{R_{\text {calib }} R_{\text {bg }}}{R_{\text {blazar }}^{2}}\right)\left(\frac{M D P_{\text {calib }}}{\Pi_{\text {upscatter,blazar }}}\right)^{2}
$$

From the above it is clear that the third case is gives an intermediate value for required observation time compared to the first two cases, as $\frac{R_{\text {calib }}}{R_{\text {blazar }}} \gg \frac{R_{\mathrm{bg}}}{R_{\text {blazar }}} \gg 1$ in the third case. The actual observation time will be within the range set by these first two limiting cases given by eqns. 5 and [6, that are independent of the background count rate. This is of course true if the background rates for the calibrator and blazar are the same or approximately the same. If the calibrator and blazar backgrounds are different, their ratio would appear in the equation 6. This would increase the maximum required observing time in the case that the background rate for the blazar is much higher than that for the calibrator source. In this case, the polarization signal from the blazar would simply be very hard to observe.

Here, we assume that the degree of high energy polarization of a blazar due to inverse-Compton scattering calculated from eqn. 2 should be measurable by the polarimeter. Therefore, we determine time required to do so given a dedicated observation of the particular blazar. We drop the subscript "blazar" in the tables.

\subsection{Inputs}

\subsubsection{Flux of the Crab in different energy ranges}

In order to scale off the calibrator source, either we need to express blazar fluxes in units of mCrab or convert the flux of the calibrator and blazar in the same units (photons $\mathrm{cm}^{-2} \mathrm{sec}^{-1}$ is used). Because of the difference between the Crab energy spectrum and the candidateblazar spectra, this conversion is energy-dependent, and so our first input is the flux of the Crab in each instrument's energy range. In order to determine the photon number flux of the calibrator source, there are two steps. First, we derive the energy flux in the polarimeter range

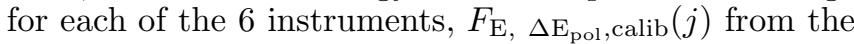

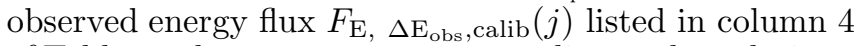
of Table 1 where $j=1-6$ corresponding to the polarimeter index. Here, $\Delta E_{\text {pol }}(j)=E_{\text {pol,min }}(j)-E_{\text {pol, } \max }(j)$ symbolizes the energy range of the polarimeter considered and $\Delta E_{\text {obs }}(j)=E_{\text {obs,min }}(j)-E_{\text {obs,max }}(j)$ symbolizes the energy range of observed fluxes used as input. And then we convert this scaled energy flux at the polarimeter energies, $F_{\mathrm{E}, \Delta \mathrm{E}_{\mathrm{pol}}, \text { calib }}(j)$ or column 5 of Table 1 into a photon flux, $F_{\mathrm{ph}, \Delta \mathrm{E}_{\mathrm{pol}}, \text { calib }}(j)$ in the same 


\begin{tabular}{|c|c|c|c|c|c|c|}
\hline $\begin{array}{c}\text { Polarimeter energy range } \\
\Delta E_{\mathrm{pol}}(j)= \\
E_{\mathrm{pol}, \min }(j)-E_{\mathrm{pol}, \max }(j) \\
(\mathrm{keV})\end{array}$ & $\begin{array}{c}\text { Observed Energy Range } \\
\Delta E_{\text {obs }}(j)= \\
E_{\text {obs,min }}(j)-E_{\text {obs, } \max }(j) \\
(\mathrm{keV})\end{array}$ & $\begin{array}{c}\text { Photon } \\
\text { index } \\
\Gamma_{\text {Crab }}(j)\end{array}$ & $\begin{array}{c}\text { Value of mCrab } \\
\text { Observed energy flux } \\
F_{\mathrm{E}}, \Delta \mathrm{E}_{\mathrm{obs}}, \operatorname{calib}(j) \\
\left(10^{-12} \mathrm{ergs} \mathrm{cm}^{-2} \mathrm{sec}^{-1}\right)\end{array}$ & $\begin{array}{c}\text { Value of mCrab } \\
\text { Scaled energy flux } \\
F_{\mathrm{E}}, \Delta \mathrm{E}_{\mathrm{pol}}, \mathrm{calib}(j) \\
\left(10^{-12} \mathrm{ergs} \mathrm{cm}^{-2} \mathrm{sec}^{-1}\right)\end{array}$ & 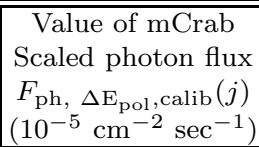 & Calibrator flux \\
\hline PoGOLITE : $25-80$ & $17-60$ & 2.1 & 14.3 & 12.8 & 19.1 & 200 \\
\hline X-Calibur : $25-80$ & $17-60$ & 2.1 & 14.3 & 12.8 & 19.1 & 25 \\
\hline GEMS-like : $2-10$ & $2-10$ & 2.1 & $2.4 \times 10^{1}$ & $2.4 \times 10^{1}$ & $3.8 \times 10^{2}$ & 1 \\
\hline POLARIX : $2-10$ & $2-10$ & 2.1 & $2.4 \times 10^{1}$ & $2.4 \times 10^{1}$ & $3.8 \times 10^{2}$ & 1 \\
\hline ASTRO-H : $50-195$ & $14-195$ & 2.1 & 23.86 & 9.151 & 6.344 & 10 \\
\hline GAP : $50-195$ & $14-195$ & 2.1 & 23.86 & 9.151 & 6.344 & 1000 \\
\hline
\end{tabular}

TABLE 1

VALUES OF MCRAB FOR THE ENERGY RANGE OF POLARIMETERS ARE LISTED HERE AND THE WAY THEY ARE OBTAINED FOR THE APPROPRIATE ENERGY RANGE FROM EXISTING DATA IS DESCRIBED SECTION 5.4.1 WE LIST THE ENERGY FLUXES IN OBSERVED ENERGY RANGES AND THE SCALED ENERGY FLUXES APPROPRIATE FOR THE POLARIMETER ENERGY RANGe, $\Delta E_{\text {pol }}(j)=E_{\text {pol,min }}(j)-E_{\text {pol,max }}(j)$, USING THE SPECTRAL index, $\Gamma_{\text {calib }}(j)$ of the Crab in the observed energy Range, $E_{\text {obs,min }}(j)-E_{\text {obs,max }}(j)$. In the final COlumn, we also list the CALIBRATOR FLUX VALUES IN MCRAB.

energy range listed in column 6 . The final column 7 , lists the calibrator flux values from column 6 in mCrab units. Since, we use observed energy fluxes as input, we chose the observed values in the energy range closest to the polarimeter range and use the photon index $\Gamma$ to scale. For the 6 polarimeters under consideration, the energy ranges are labelled as $\left(E_{\mathrm{pol}, \min }(j)-E_{\mathrm{pol}, \max }(j)\right)$. PoGOLITE and X-Calibur have $E_{\text {pol,min }}(1,2)=25$ $\mathrm{keV}$ and $E_{\text {pol,max }}(1,2)=80 \mathrm{keV}$, GEMS-like instruments and POLARIX have $E_{\text {pol,min }}(3,4)=2 \mathrm{keV}$ and $E_{\text {pol,max }}(3,4)=10 \mathrm{keV}$, and finally ASTRO-H and GAP have $E_{\mathrm{pol}, \min }(5,6)=50 \mathrm{keV}$ and $E_{\mathrm{pol}, \max }(5,6)=195$ $\mathrm{keV}$ as indicated in Table 1

IBIS / INTEGRAL measured the Crab flux in the energy range from $E_{\mathrm{obs}, \min }(1)-E_{\mathrm{obs}, \max }(1)=17-60 \mathrm{keV}$ (Krivonos et al. 2010a $)^{5}$. In order to scale to the energy range of PoGOLITE and X-Calibur, we use this flux and scale with the spectral index from SPI / INTEGRAL (Jourdain et al. 2008). For GEMS-like missions and for POLARIX we use the Crab flux measured by RXTE in the same energy range, $\Delta E_{\text {pol }}=\Delta E_{\text {obs }}=2-10 \mathrm{keV}$ , (Weisskopf et al. 2010b). This scaling is done by the following equation,

$$
\begin{aligned}
& F_{\mathrm{E}, \Delta \mathrm{E}_{\mathrm{pol}}, \mathrm{calib}}(j)=F_{\mathrm{E}, \Delta \mathrm{E}_{\mathrm{obs}}, \mathrm{calib}}(j) \\
& \times \frac{E_{\mathrm{pol}, \min }(j)^{2-\Gamma_{\text {calib }}(j)}-E_{\mathrm{pol}, \max }(j)^{2-\Gamma_{\text {calib }}(j)}}{E_{\text {obs, } \min }(j)^{2-\Gamma_{\text {calib }}(j)}-E_{\mathrm{obs}, \max }(j)^{2-\Gamma_{\text {calib }}(j)}}
\end{aligned}
$$

for $j=1-4$. $\Gamma_{\text {calib }}(j)$ is the photon index of the Crab in the considered range of energy. For $j=3,4$, the eqn. 7 trivially holds true.

For ASTRO-H and GAP $\left(\Delta E_{\mathrm{pol}}=50-195 \mathrm{keV}\right)^{6}$, we use here the Crab flux in the energy range $\Delta E_{\text {obs }}=$ $14-195 \mathrm{keV}$ from the 70 Month Swift - BAT All sky Hard X - Ray Survey (Baumgartner et al. 2013) and an average spectral index of 2.1 from several measurements (Kirsch et al. 2005). Then the Crab flux in the energy range from between the minimum energy of the observed range $E_{\mathrm{obs}, \min }(5,6)=14 \mathrm{keV}$ and the minimum

\footnotetext{
${ }^{5}$ http://heasarc.gsfc.nasa.gov/db-perl/ W3Browse/w3table.pl?tablehead=name \%3Dintibisass\&Action=More+Options

${ }^{6}$ In practice, ASTRO-H is expected to have polarimetry information from $50-300 \mathrm{keV}$, but here we will consider the range below $195 \mathrm{keV}$ where observed flux values are available.
}

polarimeter energy $E_{\mathrm{pol}, \min }(5,6)=50 \mathrm{keV}$ is derived from the IBIS flux between $E_{\mathrm{obs}, \min }(1)-E_{\mathrm{obs}, \max }(1)=$ $17-60 \mathrm{keV}$. This flux is subtracted from BAT band

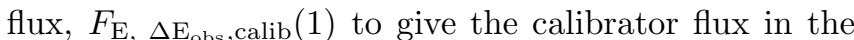
ASTRO-H / GAP energy range as,

$$
\begin{aligned}
F_{\mathrm{E}, \Delta \mathrm{E}_{\mathrm{pol}}, \text { calib }}(j) & =F_{\mathrm{E}, \Delta \mathrm{E}_{\mathrm{obs}}, \mathrm{calib}}(j)-F_{\mathrm{E}, \Delta \mathrm{E}_{\mathrm{obs}}, \mathrm{calib}}(1) \\
& \times \frac{E_{\mathrm{obs}, \min }(j)^{2-\Gamma_{\text {calib }}(1)}-E_{\mathrm{pol}, \min }(j)^{2-\Gamma_{\text {calib }}(1)}}{E_{\mathrm{obs}, \min }(1)^{2-\Gamma_{\text {calib }}(1)}-E_{\mathrm{obs}, \max }(1)^{2-\Gamma_{\text {calib }}(1)}}
\end{aligned}
$$

for $j=5,6$. Note that the numerator of the second term in the above equation, correctly has $E_{\mathrm{obs}, \min }(j)$ and $E_{\mathrm{pol}, \min }(j)$ as we want to subtract the flux in the range from $E_{\mathrm{obs}, \min }(j)-E_{\mathrm{pol}, \min }(j)=14-50 \mathrm{keV}$. For the Crab, since the spectral index is $\approx 2.1$ for both the IBIS and the BAT range one can simply use this value for both the observed and polarimeter energy ranges. For blazars, the same approach is followed with the energy range for which observed fluxes are available.

After determining the energy flux in the polarimeter energy range, the second step is to calculate the photon number flux as

$$
\begin{aligned}
F_{\mathrm{ph}, \Delta \mathrm{E}_{\mathrm{pol}}, \text { calib }}(j) & =\frac{F_{\mathrm{E}, \Delta \mathrm{E}_{\mathrm{pol}, \mathrm{calib}}(j)}}{E_{\mathrm{pol}, \min }(j)} \frac{2-\Gamma_{\text {calib }}(j)}{1-\Gamma_{\text {calib }}(j)} \\
& \times \frac{1-\left(\frac{E_{\mathrm{pol}, \max }(j)}{E_{\mathrm{pol}, \min }(j)}\right)^{1-\Gamma_{\text {calib }}(j)}}{1-\left(\frac{E_{\mathrm{pol}, \max }(j)}{E_{\mathrm{pol}, \min }(j)}\right)^{2-\Gamma_{\text {calib }}(j)}}
\end{aligned}
$$

for $j=1-6$. The Crab fluxes (both energy fluxes and photon counts) in the various energy ranges of the instruments of interest are tabulated in table 1.

\subsubsection{Fluxes, photon, and electron indices, seed polarization of blazars}

The photon fluxes of the selected blazars are computed just like for the Crab as described in the previous section. First the energy fluxes in observed ranges, are scaled to the polarimeter energy ranges with the appropriate spectral indices $\Gamma_{\text {blazar }}(j)$, as

$$
\begin{aligned}
& F_{\mathrm{E}, \Delta \mathrm{E}_{\mathrm{pol}}, \text { blazar }}(j)=F_{\mathrm{E}, \Delta \mathrm{E}_{\mathrm{obs}}, \operatorname{blazar}(j)}(j)^{2-\Gamma_{\text {blazar }}(j)} \\
& \times \frac{E_{\mathrm{pol}, \min }(j)^{2-\Gamma_{\text {blazar }}(j)}-E_{\mathrm{pol}, \max }(j)^{2-\Gamma_{\text {blazar }}(j)}}{E_{\mathrm{obs}, \min }(j)^{2-\Gamma_{\text {blazar }}(j)}-E_{\mathrm{obs}, \max }(j)^{2}}
\end{aligned}
$$




\begin{tabular}{|c|c|c|c|c|c|c|c|}
\hline $\begin{array}{c}\text { Polarimeter id } \\
\mathrm{j}\end{array}$ & \begin{tabular}{|c|} 
Polarimeter \\
energy range \\
$\Delta E_{\mathrm{pol}}(j)$ \\
$(\mathrm{keV})$ \\
\end{tabular} & \begin{tabular}{|c|} 
Energy range \\
of observed flux \\
$\Delta E_{\mathrm{obs}}(j)$ \\
$(\mathrm{keV})$ \\
\end{tabular} & 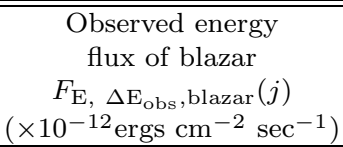 & $\begin{array}{c}\text { Scaled energy } \\
\text { flux of blazar } \\
F_{\mathrm{E}}, \Delta \mathrm{E}_{\mathrm{pol}}, \text { blazar }(j) \\
\left(\times 10^{-12} \mathrm{ergs} \mathrm{cm}^{-2} \mathrm{sec}^{-1}\right)\end{array}$ & $\begin{array}{c}\text { Photon index } \\
\text { of blazar } \\
\Gamma_{\text {blazar }}(j)\end{array}$ & $\begin{array}{c}\begin{array}{c}\text { Electron index } \\
\text { of blazar } \\
\mathrm{p}(\mathrm{j})\end{array} \\
\end{array}$ & \begin{tabular}{|c|} 
Seed \\
Polariz. degree \\
$\Pi_{\text {init }}$ \\
$(\%)$ \\
\end{tabular} \\
\hline 1,2 & $25-80$ & $17-60$ & 12.40 & 13.07 & 1.6 & 2.2 & 20.0 \\
\hline 3,4 & $2-10$ & $2-10$ & 10.00 & 10.00 & 1.56 & 2.12 & 20.0 \\
\hline$\overline{5,6}$ & $50-195$ & $14-195$ & 34.3 & 22.7 & 1.49 & 1.98 & 20.0 \\
\hline
\end{tabular}

TABLE 2

INPUT PARAMETERS FOR 3C 279 WITH DETAILED DESCRIPTION IN SECTION 5.4 .2 WE LIST THE ENERGY FLUXES IN OBSERVED ENERGY RANGES AND THE SCALED ENERGY FLUXES APPROPRIATE FOR THE POLARIMETER ENERGY RANGE, $\Delta E_{\text {pol }}(j)=E_{\text {pol,min }}(j)-E_{\text {pol,max }}(j)$,

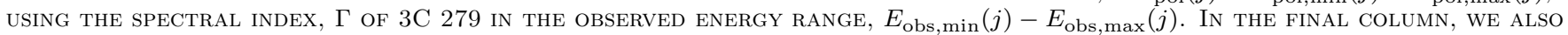
List the seed polarization degree value (temporal average). For ASTRO-H and GAP, we scale Fluxes to BAT energy RANGE OF 50-195.

\begin{tabular}{|c|c|c|c|c|c|c|c|}
\hline $\begin{array}{c}\text { Polarimeter id } \\
\mathrm{j}\end{array}$ & $\begin{array}{c}\text { Polarimeter } \\
\text { energy range } \\
\Delta E_{\mathrm{pol}}(j) \\
(\mathrm{keV})\end{array}$ & \begin{tabular}{|c|} 
Energy range \\
of observed flux \\
$\Delta E_{\text {obs }}(j)$ \\
$(\mathrm{keV})$
\end{tabular} & 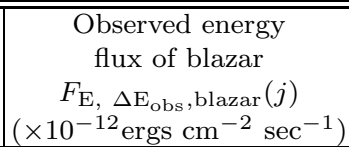 & 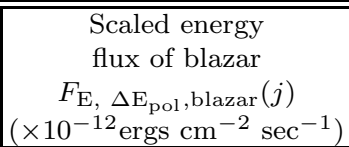 & $\begin{array}{c}\text { Photon index } \\
\text { of blazar } \\
\Gamma_{\text {blazar }}(j)\end{array}$ & $\begin{array}{c}\text { Electron index } \\
\text { of blazar } \\
\mathrm{p}(\mathrm{j})\end{array}$ & $\begin{array}{c}\text { Seed } \\
\text { Polariz. degree } \\
\Pi_{\text {init }} \\
(\%)\end{array}$ \\
\hline 1,2 & $25-80$ & $10-50$ & 38.2 & 45.7 & 1.23 & 1.46 & 15.0 \\
\hline 3,4 & $2-10$ & $2-10$ & 6.09 & 6.09 & 1.38 & 1.76 & 15.0 \\
\hline 5,6 & $50-195$ & $14-195$ & 70.0 & 36.4 & 1.38 & 1.76 & 15.0 \\
\hline
\end{tabular}

TABLE 3

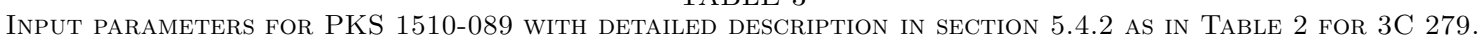

\begin{tabular}{|c|c|c|c|c|c|c|c|}
\hline \begin{tabular}{|} 
Polarimeter id \\
$\mathrm{j}$
\end{tabular} & \begin{tabular}{|c|} 
Polarimeter \\
energy range \\
$\Delta E_{\mathrm{pol}}(j)$ \\
$(\mathrm{keV})$ \\
\end{tabular} & \begin{tabular}{|c|} 
Energy range \\
of observed flux \\
$\Delta E_{\mathrm{obs}}(j)$ \\
$(\mathrm{keV})$ \\
\end{tabular} & 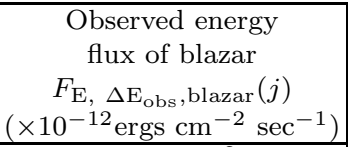 & $\begin{array}{c}\text { Scaled energy } \\
\text { flux of blazar } \\
F_{\mathrm{E}}, \Delta \mathrm{E}_{\mathrm{pol}}, \text { blazar }(j) \\
\left(\times 10^{-12} \mathrm{ergs} \mathrm{cm}^{-2} \mathrm{sec}^{-1}\right) \\
\end{array}$ & $\begin{array}{c}\text { Photon index } \\
\text { of blazar } \\
\Gamma_{\text {blazar }}(j)\end{array}$ & $\begin{array}{c}\text { Electron index } \\
\text { of blazar } \\
\mathrm{p}(\mathrm{j})\end{array}$ & \begin{tabular}{|c|} 
Seed \\
Polariz. degree \\
$\Pi_{\text {init }}$ \\
$(\%)$ \\
\end{tabular} \\
\hline 1,2 & $25-80$ & $17-60$ & $1.03 \times 10^{2}$ & $1.02 \times 10^{2}$ & 1.8 & 2.6 & 10.0 \\
\hline 3,4 & $2-10$ & $2-10$ & 17.6 & 17.6 & 1.53 & 2.06 & 10.0 \\
\hline 5,6 & $50-195$ & $14-195$ & 133.0 & 32.87 & 1.52 & 2.04 & 10.0 \\
\hline
\end{tabular}

TABLE 4

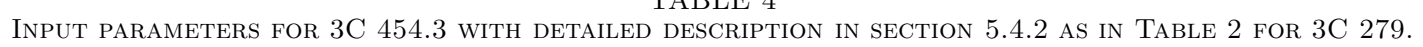

for $j=1-4$. And as in the previous section describing the calibrator flux calculation, for ASTRO-H and GAP, the energy fluxes appropriate for the polarimeter ranges need to be calculated with

$F_{\mathrm{E}, \Delta \mathrm{E}_{\mathrm{pol}}, \operatorname{blazar}}(j)=F_{\mathrm{E}, \Delta \mathrm{E}_{\mathrm{obs}}, \operatorname{blazar}}(j)-F_{\mathrm{E}, \Delta \mathrm{E}_{\mathrm{obs}}, \text { blazar }}(1)(11)$

$$
\times \frac{E_{\text {obs }, \min }(j)^{2-\Gamma_{\text {blazar }}(1)}-E_{\text {pol,min }}(j)^{2-\Gamma_{\text {blazar }}(1)}}{E_{\text {obs,min }}(1)^{2-\Gamma_{\text {blazar }}(1)}-E_{\text {obs, } \max }(1)^{2-\Gamma_{\text {blazar }}(1)}}
$$

for $j=5,6$. The fluxes and the photon indices of the blazars are taken from the literature. From the photon index, $\Gamma$, the electron index is calculated in the Thomson limit in the electron rest frame, $p=2 \Gamma-1$. This is used to calculate the high energy polarization fraction due to IC scattering, $\Pi_{e, \text { powerlaw from eqn. } 1]}$

For 3C 279, the IBIS flux from the INTEGRAL IBIS All Sky Survey (INTIBISASS) of Hard X-ray Sources 7 (Krivonos et al. 2010b) is used and scaled to PoGOLITE and X-Calibur energy ranges using the spectral index from Swift BAT AGN 60 month survey (Ajello et al. 2012). For GEMS-like and POLARIX, the mean RXTE flux from figure 4 in (Collmar et al. 2010) that is consistent with Swift XRT measurements is used. And spectral index is the Swift XRT value in (Collmar et al. 2010).

\footnotetext{
${ }^{7}$ http://heasarc.gsfc.nasa.gov/db-perl/ W3Browse/w3table.pl?tablehead=name \%3Dintibisass\&Action=More+Options
}

For ASTRO-H and GAP, the Swift BAT 70 month survey fluxes (Baumgartner et al. 2013) and Swift BAT 60 month AGN catalog indices (Ajello et al. 2012) ${ }^{8}$ are used in the range $14-195 \mathrm{keV}$. The IBIS flux for $3 \mathrm{C} 279$ in the $17-60 \mathrm{keV}$ band is then scaled to $14-50 \mathrm{keV}$ and subtracted from the BAT flux to match the appropriate range.

Similarly, fluxes and indices are determined for PKS 1510-089 and 3C 454.3. For PKS 1510-089, the fluxes taken are Suzaku (Kataoka et al. 2008) (10 - $50 \mathrm{keV})$ for PoGOLITE and X-Calibur, Swift (2 - $10 \mathrm{keV})$ (Abdo et al. 2010b) for GEMS-like and POLARIX, and the BAT 70 month survey fluxes for ASTRO-H and GAP (Baumgartner et al. 2013). Both the photon index and flux values for PKS 1510-089 are the Suzaku values in table 3 for the power-law + disk blackbody model $(P L+D B)$ model of Kataoka et al. (2008) in the 10-50 $\mathrm{keV}$ range. And for $14-195 \mathrm{keV}$, the photon index along with the flux is from Swift-BAT 70 month Hard X-ray survey (Baumgartner et al. 2013) Like for PKS 1510-089, for 3C 454.3 the fluxes are from INTIBISASS, Swift$\mathrm{XRT}$ and BAT respectively. The photon index for $3 \mathrm{C}$ 454.3 to scale to the $25-80 \mathrm{keV}$ range is taken from

\footnotetext{
${ }^{8}$ http://heasarc.gsfc.nasa.gov/db-perl/ W3Browse/w3table pl?tablehead=name \%3Dswbatagn60\&Action=More+Options
} 
combined analysis of IBIS/ISGRI, SPI and JEM-X data done in Pian et al. (2006). For $2-10 \mathrm{keV}$, the values are from are from Swift-XRT (Abdo et al. 2010b) for $2-10 \mathrm{keV}$ and for $14-195 \mathrm{keV}$, again Swift-BAT is used (Baumgartner et al. 2013). Once, we get the energy fluxes in the polarimeter energy ranges, we can readily compute the photon fluxes using the spectral indices as was done for the calibrator,

$$
\begin{aligned}
F_{\mathrm{ph}, \Delta \mathrm{E}_{\mathrm{pol}}, \text { blazar }}(j)= & \frac{F_{\mathrm{E}, \Delta \mathrm{E}_{\mathrm{pol}}, \text { blazar }}(j)}{E_{\mathrm{pol}, \min }(j)} \frac{2-\Gamma_{\mathrm{blazar}}(j)}{1-\Gamma_{\text {blazar }}(j)} \\
& \times \frac{1-\left(\frac{E_{\mathrm{pol}, \max }(j)}{E_{\mathrm{pol}, \min }(j)}\right)^{1-\Gamma_{\mathrm{blazar}}(j)}}{1-\left(\frac{E_{\mathrm{pol}, \max }(j)}{E_{\mathrm{pol}, \min }(j)}\right)^{2-\Gamma_{\mathrm{blazar}}(j)}}
\end{aligned}
$$

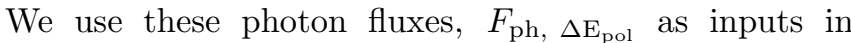
eqns. (5) and (6) instead of the source count rates, $R$. In absence of detailed energy dependent models of effective areas for every polarimeter, a consistent way of determine the photon count rates for every blazar is difficult. And hence, ignoring distinction between $F_{\mathrm{ph}, \Delta \mathrm{E}_{\mathrm{pol}}}$ and $\mathrm{R}$ we put

$$
R_{\text {blazar }}=F_{\mathrm{ph}, \Delta \mathrm{E}_{\mathrm{pol}}, \text { blazar }} ; R_{\text {calib }}=F_{\mathrm{ph}, \Delta \mathrm{E}_{\mathrm{pol}}, \text { calib }}
$$

This difference is only important for those blazars whose photon indices are different from the average Crab index of 2.1. An approximate calculation for ASTRO-H assuming a power-law dependence of effective area on energy 9 shows that for PKS 1510-089 with the most different spectral index of $\approx 1.4$, there is approximately a factor of 2 difference in the ratio of $R_{\text {blazar }} / R_{\text {calib }}$ when the energy dependence of the effective area is accounted for. Thus, it is not a significant difference in the observation times relative to the range calculated here.

Seed photon IR polarization values are taken from average values consistent with Fuiiwara et al. (2012); Ikejiri et al. (2011). All these inputs are listed in Tables (2), (3), (4).

\subsection{Polarimeter sensitivities \& polarization detection prospects}

\subsubsection{Detection times for the polarimeters}

The polarimeter sensitivities are expressed in terms of their ability to detect polarization from a Crab-like signal quantified in terms of the minimum detectable polarization in Eq. (4). The high-energy polarization degree for each test source is derived from Eq. 1 using seed photon IR polarization values from Ikejiri et al. (2011); Fujiwara et al. (2012), and, together with the other inputs discussed above, can be used to compute the exposure or observation time, $T_{\text {obs blazar }}$ required to detect the polarization degree from the blazar under consideration.

As an illustration we look at the case of polarimetry with INTEGRAL. Both main instruments on board INTEGRAL, IBIS and SPI, have reported polarization measurements of GRBs (Götz et al. 2013, 2009; McGlynn et al. 2007, etc.,). Thus, it is natural to check prospects of blazar measurements with INTEGRAL. With a modulation factor

${ }^{9}$ http://astro-h.isas.jaxa.jp/researchers/sim/ effective_area.html of $\sim 20 \%$ Kalemci et al. (2004) in the spectrometer on INTEGRAL, SPI, for the Crab, with $R_{S}=$ 0.18 counts sec${ }^{-1}$ and $R_{\mathrm{bg}}=14$ counts sec${ }^{-1}$, an exposure of $1000 \mathrm{ksec}$ gives $M D P=44.4 \%$. 3C454.3, one of the brightest blazars which is also known to flare, has a flux of $1.85 \times 10^{-10} \mathrm{ergs} \mathrm{cm}^{-2} \mathrm{sec}^{-1}$ (Vercellone \& for the AGILE Team 2012). Scaling off the Crab using Eq. (4), this translates into a detection time of $\approx 10^{8} \mathrm{ksec}$. Therefore, this simple calculation shows that INTEGRAL is not well-suited to detect polarization from blazars.

For other high-energy polarimeters, we determine the observation times using eqns. (5) and (6). Tables 5 56/7/819]10 show approximate observation times required to detect polarization of 3C 279, PKS 1510-089 and 3C 454.3 using the polarimeters, PoGOLITE, X-Calibur, GEMS-like missions, POLARIX, ASTRO-H and GAP. The scaling is done in terms of the photon number fluxes from observed energy fluxes. To do this computation, the effective areas for each polarimeter must be used to determine the counting rates, $\mathrm{R}$ for both $\mathrm{Crab}$ and the blazars. However, it is difficult to do this for future missions where the energy dependence of the effective areas is not understood very well yet. As described in the previous section, for our candidate blazars, this difference results in a factor of order unity, which is less than the factor between the background and signal dominated regimes. The last column indicates the exposure times required to jointly measure the same polarization degree, $\Pi$ and position angle, $\psi$ at $3 \sigma$ above zero in accordance with Weisskopf et al. (2010a); Strohmayer \& Kallman (2013). The times increase in proportion to the square of the significance of the measurement. Thus, in going from a 3-sigma measurement to a 5-sigma measurement, the required time will increase by a factor of $\approx 2.8$ for a measurement of either 1 or 2 parameters. In going from a 3 -sigma 1 parameter measurement to a 5 -sigma 2 parameter measurement, this factor is $\left(\frac{3^{2}}{4}\right) \times\left(\frac{5}{3}\right)^{2}=6.25$ All these times are subject to the assumption that systematic errors do not dominate the statistical errors.

\subsubsection{Detection prospects and strategy}

From these estimates it is clear that the detection of polarization from blazars is challenging with balloonborne polarimeters compared to space polarimeters. Space based instruments will almost certainly detect a signal, if indeed the high energy emission from jets in blazars is polarized. On the other hand, balloon-borne experiments with a higher detection time (equivalently a higher MDP threshold) will need to have their systematics moderated and perhaps need blazars with a higher flux and degree to find a positive detection. Flaring blazars observed with dedicated balloon flights may have a chance of having their polarization detected. This will demand planned observations based on triggers from the optical, UV, IR telescopes monitoring flaring activity. Our estimates based on the MDP (Guo et al. 2013; Pearce et al. 2012) suggest X-Calibur will have a better chance of detecting a polarization signal from blazars than PoGOLITE.

With the future space based instruments, the sensitivity is much higher and thus statistically significant detections are highly likely with reasonable observing times. 


\begin{tabular}{|c|c|c|c|c|c|c|}
\hline \hline Source & $\begin{array}{c}\text { Photon Flux } \\
\text { Sensitivity }\left(R_{\text {calib }}\right) \\
\left(\times 10^{-5} \mathrm{~cm}^{-2} \mathrm{sec}^{-1}\right)\end{array}$ & $\begin{array}{c}\text { MDP } \\
(\%)\end{array}$ & $\begin{array}{c}\text { Photon Flux } \\
\left(R_{\text {blazar }}\right) \\
\left(\times 10^{-5} \mathrm{~cm}^{-2} \mathrm{sec}^{-1}\right)\end{array}$ & $\begin{array}{c}\text { High Energy } \\
\text { Polariz. Degree } \\
\Pi_{\text {upscatter }}(\%)\end{array}$ & $\begin{array}{c}3-\sigma \Pi \\
\text { measurement time } \\
\frac{T_{\text {obs,blazar }}}{T_{\text {obs,calib }}}\end{array}$ & $\begin{array}{c}3-\sigma(\Pi, \psi) \\
\text { measurement time } \\
\frac{n_{\sigma}^{2}}{4} \frac{T_{\text {obs,blazar }}}{T_{\text {obs,calib }}}\end{array}$ \\
\hline 3C 279 & $3.82 \times 10^{3}$ & 10.0 & 18.50 & 10.81 & $1.77 \times 10^{2}-3.65 \times 10^{4}$ & $3.98 \times 10^{2}-8.21 \times 10^{4}$ \\
\hline PKS 1510-089 & & & 62.0 & 6.94 & $(1.28-78.8) \times 10^{2}$ & $2.88 \times 10^{2}-1.78 \times 10^{4}$ \\
\hline 3C 454.3 & & & $1.47 \times 10^{2}$ & 5.73 & $7.92 \times 10^{1}-2.06 \times 10^{2}$ & $1.78 \times 10^{2}-4.63 \times 10^{3}$ \\
\hline
\end{tabular}

TABLE 5

ObSERVATIONAl PRospects For PoGOLITE (25-80 keV): 200 mCrab source at $10 \%$ polarization detectable in 1 flight (20 days). Measurement times calculated using eqns 5 and 6 ]

\begin{tabular}{|c|c|c|c|c|c|c|}
\hline \hline Source & $\begin{array}{c}\text { Photon Flux } \\
\text { Sensitivity }\left(R_{\text {calib }}\right) \\
\left(\times 10^{-5} \mathrm{~cm}^{-2} \mathrm{sec}^{-1}\right)\end{array}$ & $\begin{array}{c}\text { MDP } \\
(\%)\end{array}$ & $\begin{array}{c}\text { Photon Flux } \\
\left(R_{\text {blazar }}\right) \\
\left(\times 10^{-5} \mathrm{~cm}^{-2} \mathrm{sec}^{-1}\right)\end{array}$ & $\begin{array}{c}\text { High Energy } \\
\text { Polariz. Degree } \\
\Pi_{\text {upscatter }}(\%)\end{array}$ & $\begin{array}{c}3-\sigma \Pi \\
\text { measurement time } \\
\frac{T_{\text {obs,blazar }}}{T_{\text {obs,calib }}}\end{array}$ & $\begin{array}{c}3-\sigma, \psi) \\
\text { measurement time } \\
\frac{n_{\sigma}^{2}}{4} \frac{T_{\text {obs, blazar }}}{T_{\text {obs,calib }}}\end{array}$ \\
\hline 3C 279 & $4.78 \times 10^{2}$ & 4.5 & 18.50 & 10.81 & $4.47-115.39$ & $10.07-2.60 \times 10^{2}$ \\
\hline PKS 1510-089 & & & 62.0 & 6.94 & $3.24-25.0$ & $7.29-56.2$ \\
\hline 3C 454.3 & & & $1.47 \times 10^{2}$ & 5.73 & $2.00-6.52$ & $4.51-14.7$ \\
\hline
\end{tabular}

TABLE 6

ObSERVATIONAL PRospects For X-CALibur $(25-80 \mathrm{keV}): 25$ mCrab source at $4.5 \%$ polarization is detectable in $1000 \mathrm{ksec}$. Measurement times calculated using eqns 5 and 6

\begin{tabular}{|c|c|c|c|c|c|c|}
\hline \hline Source & $\begin{array}{c}\text { Photon Flux } \\
\text { Sensitivity }\left(R_{\text {calib }}\right) \\
\left(\times 10^{-5} \mathrm{~cm}^{-2} \mathrm{sec}^{-1}\right)\end{array}$ & $\begin{array}{c}\text { MDP } \\
(\%)\end{array}$ & $\begin{array}{c}\text { Photon Flux } \\
\left(R_{\text {blazar }}\right) \\
\left(\times 10^{-5} \mathrm{~cm}^{-2} \mathrm{sec}^{-1}\right)\end{array}$ & $\begin{array}{c}\text { High Energy } \\
\text { Polariz. Degree } \\
\Pi_{\text {upscatter }}(\%)\end{array}$ & $\begin{array}{c}3-\sigma \text { - } \\
\text { measurement time } \\
\frac{T_{\text {obs, blazar }}}{T_{\text {obs,calib }}}\end{array}$ & $\begin{array}{c}3-\sigma(\Pi, \psi) \\
\text { measurement time } \\
\frac{n_{\sigma}^{2}}{4} \frac{T_{\text {obs, blazar }}}{T_{\text {obs,calib }}}\end{array}$ \\
\hline 3C 279 & $3.8 \times 10^{2}$ & 2.0 & 142.0 & 10.66 & $9.4 \times 10^{-2}-2.5 \times 10^{-1}$ & $(2.1-5.7) \times 10^{-1}$ \\
\hline PKS 1510-089 & & & 83.0 & 7.46 & $0.3-1.5$ & $7.4 \times 10^{-1}-3.4$ \\
\hline 3C 454.3 & & $2.48 \times 10^{2}$ & 5.28 & $2.2 \times 10^{-1}-3.4 \times 10^{-1}$ & $(5.0-7.6) \times 10^{-1}$ \\
\hline
\end{tabular}

TABLE 7

ObSERVATIONAL PRospects For GEMS-Like instrument $(2-10 \mathrm{KEV}): 1$ MCRAB SOURCE

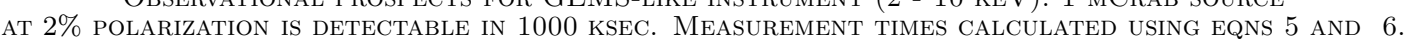

\begin{tabular}{|c|c|c|c|c|c|c|}
\hline \hline Source & $\begin{array}{c}\text { Photon Flux } \\
\text { Sensitivity }\left(R_{\text {calib }}\right) \\
\left(\times 10^{-5} \mathrm{~cm}^{-2} \mathrm{sec}^{-1}\right)\end{array}$ & $\begin{array}{c}\text { MDP } \\
(\%)\end{array}$ & $\begin{array}{c}\text { Photon Flux } \\
\left(R_{\text {blazar }}\right) \\
\left(\times 10^{-5} \mathrm{~cm}^{-2} \mathrm{sec}^{-1}\right)\end{array}$ & $\begin{array}{c}\text { High Energy } \\
\text { Polariz. Degree } \\
\Pi_{\text {upscatter }}(\%)\end{array}$ & $\begin{array}{c}3-\sigma \Pi \\
\text { measurement time } \\
\frac{T_{\text {obs, blazar }}}{T_{\text {obs,calib }}}\end{array}$ & $\begin{array}{c}3-\sigma(\Pi, \psi) \\
\text { measurement time } \\
\frac{n_{\sigma}^{2}}{4} \frac{T_{\text {obs, blazar }}}{T_{\text {obs,calib }}}\end{array}$ \\
\hline 3C 279 & $3.8 \times 10^{2}$ & 10.0 & 142.0 & 10.66 & $2.4-6.3$ & $5.3 \times 10^{0}-1.4 \times 10^{1}$ \\
\hline PKS 1510-089 & & & 83.0 & 7.46 & $8.2 \times 10^{0}-3.8 \times 10^{1}$ & $(1.9-8.5) \times 10^{1}$ \\
\hline 3C 454.3 & & & $2.48 \times 10^{2}$ & 5.28 & $5.5-8.4$ & $1.2 \times 10^{1}-1.9 \times 10^{1}$ \\
\hline
\end{tabular}

TABLE 8

OBSERVATION PROSPECTS FOR POLARIX (2.0-10.0 KEV): 1 MCRAB SOURCE AT

$10 \%$ polarization is detectable in 100 KSec. Measurement times Calculated using eqns 5 and 6

\begin{tabular}{|c|c|c|c|c|c|c|}
\hline \hline Source & $\begin{array}{c}\text { Photon Flux } \\
\text { Sensitivity }\left(R_{\text {calib }}\right) \\
\left(\times 10^{-5} \mathrm{~cm}^{-2} \mathrm{sec}^{-1}\right)\end{array}$ & $\begin{array}{c}\text { MDP } \\
(\%)\end{array}$ & $\begin{array}{c}\text { Photon Flux } \\
\left(R_{\text {blazar }}\right) \\
\left(\times 10^{-5} \mathrm{~cm}^{-2} \mathrm{sec}^{-1}\right)\end{array}$ & $\begin{array}{c}\text { High Energy } \\
\text { Polariz. Degree } \\
\Pi_{\text {upscatter }}(\%)\end{array}$ & $\begin{array}{c}3-\sigma \Pi \\
\text { measurement time } \\
\frac{T_{\text {obs, blazar }}}{T_{\text {obs,calib }}}\end{array}$ & $\begin{array}{c}3-\sigma(\Pi, \psi) \\
\text { measurement time } \\
\frac{n_{\sigma}^{2}}{4} \frac{T_{\text {obs, blazar }}}{T_{\mathrm{obs}, \text { calib }}}\end{array}$ \\
\hline 3C 279 & $6.344 \times 10^{1}$ & 4.3 & 14.3 & 10.40 & $7.58 \times 10^{-1}-3.37$ & $1.71-7.57$ \\
\hline PKS 1510-089 & & & 22.6 & 7.46 & $0.93-2.62$ & $2.10-5.89$ \\
\hline 3C 454.3 & & 20.61 & 5.26 & $2.06-6.33$ & $4.59-14.0$ \\
\hline
\end{tabular}

TABLE 9

Observational Prospects For ASTRO-H (50-195 KeV): 10 mCrab

Source At $4.3 \%$ is Detectable in 1000 KSEC. Measurement times Calculated using eqns 5 and 6

Space based instruments are not dedicated merely to polarization measurements and therefore, observational strategies are required. Due to the high sensitivity, quiescent or non-flaring blazars are also expected to be discovered. This is achievable during all sky scans as well as by targeted, longer observations of the more dim blazars.
And for flaring blazars optical, UV, IR triggers can be used for making detections. These strategies must be kept in mind while planning for future missions.

\subsection{Motivation for synchronous multiwavelength polarimetry}




\begin{tabular}{|c|c|c|c|c|c|c|}
\hline \hline Source & $\begin{array}{c}\text { Photon Flux } \\
\text { Sensitivity }\left(R_{\text {calib }}\right) \\
\left(\times 10^{-5} \mathrm{~cm}^{-2} \mathrm{sec}^{-1}\right)\end{array}$ & $\begin{array}{c}\text { MDP } \\
(\%)\end{array}$ & $\begin{array}{c}\text { Photon Flux } \\
\left(R_{\text {blazar }}\right) \\
\left(\times 10^{-5} \mathrm{~cm}^{-2} \mathrm{sec}^{-1}\right)\end{array}$ & $\begin{array}{c}\text { High Energy } \\
\text { Polariz. Degree } \\
\Pi_{\text {upscatter }}(\%)\end{array}$ & $\begin{array}{c}3-\sigma \Pi \\
\text { measurement time } \\
\frac{T_{\text {obs,blazar }}}{T_{\text {obs, calib }}}\end{array}$ & $\begin{array}{c}3-\sigma(\Pi, \psi) \\
\text { measurement time } \\
\frac{n_{\sigma}^{2}}{4} \frac{T_{\text {obs, blazar }}}{T_{\text {obs, calib }}}\end{array}$ \\
\hline 3C 279 & $6.344 \times 10^{3}$ & 20.0 & 14.3 & 10.40 & $1.64 \times 10^{3}-7.28 \times 10^{5}$ & $3.69 \times 10^{3}-1.64 \times 10^{6}$ \\
\hline PKS 1510-089 & & & 22.6 & 7.46 & $2.02 \times 10^{3}-5.66 \times 10^{5}$ & $4.54 \times 10^{3}-1.27 \times 10^{6}$ \\
\hline 3C 454.3 & & & 20.61 & 5.26 & $4.41 \times 10^{3}-1.37 \times 10^{6}$ & $1.00 \times 10^{4}-3.03 \times 10^{6}$ \\
\hline
\end{tabular}

TABLE 10

ObServational PROSPECTS FOR GAP (50.0-300.0 KeV): 1 CRAB SOURCE AT 20\%

polarization is Detectable in 2 days. Measurement times Calculated using eqns 5 and 6

In addition to using published and archival data, it would be highly effective in to perform synchronous, multiwavelength polarimetry with high-energy polarimeters and low energy polarimeters suited for blazar observations, such as RoboPol (?), F-GAMMA (Angelakis et al. 2010), and OVRO (Richards et al. 2011). From the above results, idea of using a dedicated, specialised polarimeter at longer wavelengths in conjunction with a high energy polarimeter will significantly improve the chances of detecting high energy polarization from blazars. This is because monitoring the flaring activity of blazars as described in detail the next section can be potentially be very useful to improve detection prospects. Furthermore, there is evidence that polarization certainly at low energies is dynamic (?). This suggests that given low energy polarization is ultimately a source of high energy polarization, in the SSC scenario, polarimetric variability could be crucial at X-rays and gamma rays too. Polarimetric variability means that there are times when the degree of polarization increases and therefore detectability improves (Sasada et al.|2011). In cases where the amount of polarization decreases with flux, variability in flux could yet be connected to that in polarization (Ikejiri et al. 2011; Abdo et al. 2010a), a connection that would ultimately lead to clues about the underlying mechanisms. Thus, multiwavelength polarimetry sharpen the emission models which connect the low energy to the high energy emission. Therefore, in order to faithfully probe the emission models, it is important to perform synchronised multiwavelength observations. Dedicated blazar polarimetric monitoring programs (such as RoboPol) are thus critical to our understanding of polarization and in general emission processes in blazars.

\section{CHANGE IN DETECTABILITY DUE TO FLARING}

\subsection{Different effects of flaring}

Blazars are well-known to flare at all observed wavelengths, including in the gamma-ray (e.g., Nalewajko 2013, and references therein). The high energy (X-ray and gamma-ray) fluxes can vary by up to a factor of 10 or 20 when they flare (e.g., Nalewajko 2013, and references therein). Of course, the factors could be higher for exceptional flares. Even the low energy fluxes have high flaring factors (Ulrich et al. 1997). As discussed in the previous section, flaring activity of blazars affect the detectability in the polarization domain. The direct reason is simply that those blazars in their quiescent state falling below the detection sensitivity of high energy polarimeters, may be pushed above threshold when they flare. In practice however, from the fluxes of our candi- date blazars in the previous section and the observation times needed to detect them, it is only the very bright few blazars that will be detected anyway. And so the bright end of the source flux distribution $\frac{d N}{d F}$ is of interest. This is what we discuss in the next section 6.2

Secondly, the polarization signal itself may vary due to the flaring activity. This could reduce the detectability if the degree of polarization goes down, as in the case of the gamma-ray flare seen from 3C 279 (Abdo et al. 2010a). Detectability also could be reduced to due to variation in the polarization angle (Marscher et al. 2008; Abdo et al. 2010a). Alternately, the detectability could improve due to a positive correlation between flux and polarization, as reported in the case of AO $0235+164$ and PKS 1510-089 by Sasada et al. (2011). This effect needs to be studied observationally. However, as shown in Ikeiiri et al. (2011), there is no statistically significant correlation between the variation in optical and nearIR flux and variation in polarization properties namely polarization degree, $\Pi$ and position angle, $\psi$ of flaring blazars. Only $30 \%$ blazars in their well observed sample showed a positive correlation in the flux and polarization degree variations and $12 \%$ a negative correlation. And only 3-4 blazars showed rotation of the polarization plane, with flaring. Also, there is no established statistical correlation between variations in gamma-ray flux and polarization properties. We will quantify the effect on an individual blazar that shows some correlation, later in this section. However, this is not a significant effect for a statistical sample of blazars. Therefore our treatment will ignore this effect.

\subsection{Quantitative effect of flux increase on detectability}

Here we quantify the increase in the detected fraction of blazars purely due to an increase in their flux. This effectively amounts to lowering of the detection limit by the same factor. For this we use the formalism of Feldman and Pavlidou (in preparation) to compute this increased detection fraction detailed in Appendix $\mathrm{A}$ This formalism models blazar high-energy output with two states: a quiescent state with flux $F_{\mathrm{q}}$, and a flaring state with flux $F_{\mathrm{f}}=\eta_{\text {flare }} F_{\mathrm{q}}$, where the flaring enhancement factor is typically $\eta_{\text {flare }} \sim 10$. The duty cycle $\chi$ gives the fraction of time spent in the flaring state, with typical values $\sim 20 \%$. This means that the time-averaged mean flux $F=\left[1+\left(\eta_{\text {flare }}-1\right) \chi\right] F_{\mathrm{q}}$ is higher than the quiescent flux $F_{\mathrm{q}}$. Picking an average value of the flaring factor and duty cycle for the population of blazars is an assumption which can easily be violated depending on the energy band of observation, the chosen blazar and indeed at different times for a single blazar. There is a large variance 
in flare properties of blazars and it is beyond the scope of this paper to delve into any of these complications. However, one can still make conservative predictions of increase in detectability of blazars by picking conservative values of flare factors and duty cycle. Higher flare factors and lower duty cycles will result in a positive increase in the detectability.

As mentioned before, polarization is likely to be detectable only in the brightest blazars, and thus we study the effects of flaring on the bright end of the source flux distribution. The LAT distribution (Abdo et al. 2010c of blazar fluxes shows that the the number of sources above a mean flux $F$ is a broken power law, with the two regimes divided at a "break" flux, $F_{\mathrm{b}}$. The brightest blazars have $F>F_{\mathrm{b}}$. In this regime, the observed flux distribution is $d N / d F \propto F^{-\beta_{1}}$ where $\beta_{1}=2.49$, when the entire sample of blazars (i.e. both FSRQs and BL LACs) is taken. Thus, the number of blazars detected by a high energy polarimeter in their quiescent state is

$$
N_{\text {det }}=\int_{\mathrm{F}_{\text {sens }}}^{\infty} d F \frac{d N}{d F} \propto\left(\frac{F_{\text {sens }}}{F_{\mathrm{b}}}\right)^{1-\beta_{1}}
$$

when the flux sensitivity is $F_{\text {sens }}$ of the polarimeter.

Now the effect of flaring is to effectively reduce the sensitivity limit by the flaring factor, $\eta_{\text {flare }}$ from $F_{\text {sens }}$ to $F_{\text {sens }} / \eta_{\text {flare }}$. For a typical flaring factor $\eta_{\text {flare }} \sim 10$, one would expect the number of sources to increase by a factor of $\eta_{\text {flare }}^{\beta_{1}-1}$, or $\approx 1.5$ orders of magnitude. On the other hand, the enhancement is only present for a fraction of time given by the duty cycle. This implies that effectively, one does not reduce the sensitivity by the full flaring factor $\eta_{\text {flare }}$, but instead reduces the sensitivity by a lower valued effective factor $\eta_{\text {flare,eff }}$ weighted by the duty cycle as given by Eq. A3 . This reduces the total number of sources detected when flaring to,

$$
N_{\mathrm{f}, \mathrm{det}}=\int_{\mathrm{F}_{\mathrm{sens}} / \eta_{\mathrm{flare}, \mathrm{eff}}}^{\infty} d F \frac{d N}{d F} \propto\left(\frac{F_{\mathrm{sens}} / \eta_{\text {flare }, \mathrm{eff}}}{F_{\mathrm{b}}}\right)^{1-\beta_{1}}
$$

For values, $\chi=20 \%$ and $\eta_{\text {flare }}=10, \eta_{\text {flare,eff }}=3.57$ which gives an increase factor of 6.67 instead of 1.5 orders of magnitude. This implies that the number of additional sources detected above the sensitivity threshold due to flaring is $\approx 5-6$ times the number if the sources were in their quiescent state. This is of course assuming that all sources can in principle flare and that the observation time is long enough all of these flares have been observed. At any given instant, the number of additional sources is simply given by the product of the number of additional sources overall, and the duty cycle $\chi$. Thus, the instantaneous boost for a duty cycle of $20 \%$ is 1 additional source for every quiescent source.

As there are a handful of blazars at the bright end of the source flux distribution to begin with, instead of a detailed statistical description, individual blazars that are known to flare and have a high degree of polarization at the lower energies should be selected for pointed observations. Individually, some blazars such as 3C 454.3 do show some correlation in the variation of their polarization properties with flaring. 3C 454.3 for instance has exhibited a rotation in polarization angle with flaring. The reduction in the measured polarization degree could be as high as a factor of 100 from Table 2 in Ikejiri et al. (2011) with a variation from $0.2-22.5 \%$. The variation in flux is also nearly a factor of 10 in V-band magnitudes. There is evidence for correlation over a day timescale between X-ray and gamma ray variability on one hand and optical variability on the other (Jorstad et al. 2010). Thus, if the high energy fluxes also vary proportionally, the amplification in high energy flux is also a factor of 10 . Scaling from Eq. 4. this could lead a factor of $\approx 25-250$ increase in the detection time, if there is perfect anticorrelation. On the other hand, given the nature of correlation found in Ikejiri et al. (2011), this is quite likely not such a drastic effect, and could even in some phases reduce the detection time.

\section{DISCUSSION AND CONCLUSIONS}

The prospects of detecting high energy polarization from blazars appear to be very realistic with current and future generations of polarimeters. The space based detectors such as POLARIX, ASTRO-H and a GEMS-like mission are quite likely to detect a polarization signal from blazars within reasonable observation times, based on their sensitivities, if the systematic uncertainties are not dominant. ASTRO-H for instance, could detect the listed blazars in $\sim 10^{6}$ seconds with a modest degree of polarization $O(10 \%)$, and fluxes in a state of quiescence. A GEMS-like mission will take a few hours to do this. POLARIX has the potential to do the same in less than an hour. GAP is specifically designed for GRBs, however, it is quite possible that a flaring blazar whose polarization does not reduce with flux may be detected by it. Balloon borne polarimeters cannot do as well. Between the two listed here, X-Calibur is relatively the more sensitive, though our predictions suggest it will be challenging for it to provide a detection in reasonable exposure times. The key would be to observe a variable source with a positive correlation between flux and polarization degree in an episode of flaring.

These observing prospects quantified in terms of the observation times required to make these detections are based on the MDP expression in Eq. 4. The underlying assumption is that the measurements are not limited by systematics and other observational constraints like mode of observation and high backgrounds near the source region. Therefore, the observation times may increase depending on these factors. However, our results provide an indication of the promise of blazar detections with several polarimeters particularly space based and the comparisons between them. Furthermore, we make the distinction between time required to measure the polarization degree alone from these blazars and that taken to produce joint measurements of the degree and the position angle Weisskopf et al. (2010a); Strohmaver \& Kallman (2013). While a positive detection of a polarization signal from blazars will be a big achievement in adding a new source class to high energy polarimetry, a measurement of the degree will ultimately put constraints on the physics of jet emission.

As described in section (6), variability of blazar fluxes has a key role to play in the detection prospects. Statistically, the number of blazar detections are likely to increase with flaring events, possibly by a factor of 5-6 if we wait long enough allowing for all flaring candidates to flare. The instantaneous increase in sources on the other hand is $\approx 100 \%$ for a duty cycle of $20 \%$ or 1 additional blazar per quiescent blazar already detected. This 
is purely accounting for flux increase due to flaring. In practice, since the brightest blazars at the tail end of the flux distribution are the ones that are most likely to overcome the polarization detection threshold, this leads to a handful of additional blazars. Therefore, the detection strategy would be to plan observations of the brightest blazars with a history of flaring. A further selection can be made from these depending upon correlation between low energy polarization variations and high energy flux variability. Individual blazars, show an increase or reduction in detectability depending on variation of polarization properties with that in flux. 3C 454.3 for instance, may be more challenging to detect in polarization due to rotation of the polarization angle with flaring. Examples like this and their counterexamples strongly emphasise the need for synchronous, multiwavelength polarimetric observations. For this reason dedicated high-cadence optopolarimetric programs optimized for blazar detection are crucial to such observations. In general, the success of pointed blazar observations by high energy polarimeters could lie in the wealth of polarimetric and variability information provided observational studies at lower energies. Such observations will help select the most promising targets for blazar observations and also the times when they are most likely to be detectable based on variability.

Not just episodic increases in flux, but persistent variability is also connected to polarimetry. A quantitative understanding of multiwavelength variability of blazars using statistical tools to determine timescales, correlation properties of variability constrains not only the size and location of the emission region, but also emission mechanisms, magnetic fields etc. Thus, variability and polarimetric studies are complementary and should go hand in hand.

Polarimetry represents the next frontier in high energy astronomy with several scientific questions hinging on it. As indicated by several authors (e.g... Krawczynski 2012; Krawczynski et al. 2013; Zhang \& Böttcher 2013), polarization can help to distinguish between different emission mechanisms of jets i.e. leptonic vs hadronic and also between different leptonic models. It is complementary to (non) detection of neutrinos from blazars. The magnetic field structure is directly probed by polarimetry (Krawczynski 2012; Zhang \& Böttcher 2013). Measurements of high energy polarimetry are thus, a powerful probe of several astrophysical and physical questions.

We would like to thank Felix Aharonian, Paolo Coppi, Markus Boettcher, Hirokazu Odaka for useful discussions on prospects of high energy polarimetry in general. We would also like to thank the anonymous referee for a detailed critical review that helped improve the paper. NC acknowledges support from his host institution, MPIK. VP acknowledges support by the "RoboPol" project, which is implemented under the "Aristeia" Action of the "Operational Programme Education and Lifelong Learning" and is co-funded by the European Social Fund (ESF) and Greek National Resources, and by the European Comission Seventh Framework Programme (FP7) through grants PCIG10-GA-2011-304001 "JetPop" and PIRSES-GA-2012-31578 "EuroCal". The work of BDF was partially supported by NASA via the Astrophysics Theory Program through award NNX10AC86G.

\section{APPENDIX}

\section{EFFECT OF FLUX INCREASE DUE TO FLARING ON SOURCE FLUXES}

Every blazar flares given a long enough time. Feldman and Pavlidou treat the flaring and quiescent blazars as a single population with two states. Both flaring and quiescent blazars are represented by a single luminosity function. The two states differ by a flaring factor is given by

$$
\eta_{\text {flare }}=\frac{F_{\mathrm{f}}}{F_{\mathrm{q}}}
$$

where $F_{\mathrm{f}}$ and $F_{\mathrm{q}}$ are fluxes in the flaring and quiescent state respectively. This flaring factor is assumed to be the same for all blazars and uniform in flux. This is however an assumption that is very often untrue as there is substantial variance in the flare properties. Flare amplitudes may vary from a factor of a few to nearly 3-4 orders of magnitude in exceptional cases (Feldman and Pavlidou in preparation). This depends on the energy band of observation. Also, the same blazar can have a variation in the duty cycle and flaring amplitude (e.g., Jorstad et al. 2010). Our goal is not to delve into these details, but make some conservative estimates of the increase in detectability of blazars based on average properties.

Feldman and Pavlidou (in preparation) find that the source flux distribution fitting the Fermi -LAT data has contributions from both quiescent and flaring sources (Abdo et al. 2010c). This is at GeV energies. We need an extrapolation of the distribution down to the fluxes at $\mathrm{keV}$ and $\mathrm{MeV}$ energies at which the polarimeters operate in absence of observed source flux distribution at these energies. In practice, this is easy as blazars have a power-law spectrum. And this will be done at the end when we provide numbers for the fractional increase in the detected blazars.

As a result of flaring, the break flux $F_{\mathrm{b}}$ in the source flux distribution as observed in Fermi -LAT is shifted to the lower value $F_{\mathrm{b}} / \eta_{\text {flare }}$. So, the source flux distribution is modified to include both flaring and quiescent blazars as they may be treated as a single population given by,

$$
\left.\frac{d N}{d F}\right|_{\mathrm{obs}}=A\left(\frac{F}{F_{\mathrm{b}}}\right)^{-\beta_{1}}+A \eta_{\text {flare }} C_{\mathrm{q}}\left(\frac{\eta_{\text {flare }} F}{F_{\mathrm{b}}}\right)^{-\beta_{1}}, F \geq F_{\mathrm{b}}
$$

since, we are only interested in the high end of the blazar flux distribution. Here $C_{\mathrm{q}}$ represents the factor by which the number of blazars in the quiescent state exceed the number in the flaring state at any given time in the universe 
and is related to the fraction of the times the blazar is in the high state or the duty cycle, $\chi$ as $C_{\mathrm{q}}=\frac{1-\chi}{\chi}$. In this case, the flaring flux $F_{\mathrm{f}}$ is related to the mean flux, $\langle F\rangle$ as,

$$
F_{\mathrm{f}}=\frac{\eta_{\text {flare }}}{\left(\eta_{\text {flare }}-1\right) \chi+1}\langle F\rangle=\eta_{\text {flare }, \text { eff }}\langle F\rangle
$$

The mean flux is computed for the limit when all additional blazars that exceed the sensitivity limit due to flaring, are detected. For a duty cycle, $\chi=20 \%$ and a flaring factor, $\eta_{\text {flare }}=10$, the effective flaring factor is $\eta_{\text {flare,eff }}=3.57$.

Suppose, $N_{\mathrm{u}}$ is the number of sources undetected in the quiescent state, but that are detected during their flaring state. Therefore,

$$
\begin{aligned}
N_{\mathrm{u}}=\int_{\frac{F_{\text {sens }}}{\eta_{\mathrm{flare}, \mathrm{eff}}}}^{F_{\text {sens }}} \frac{d N}{d F} d F & =\int_{\frac{F_{\text {sens }}}{\eta_{\text {flare } \mathrm{eff}}}}^{\infty} \frac{d N}{d F} d F-\int_{\frac{F_{\text {sens }}}{\eta_{\mathrm{flare}}}}^{\infty} \frac{d N}{d F} d F \\
& =N_{\mathrm{f}, \mathrm{det}}-N_{\mathrm{q}, \mathrm{det}}
\end{aligned}
$$

where $F_{\text {sens }}$ is the sensitivity of the polarimeter and $\eta_{\text {flare,eff }}$ is the effective amplication factor due to flaring accounting for a finite duty cycle. Therefore the fractional increase of sources observed due to flaring increases is given by,

$$
f=\frac{N_{\mathrm{u}}}{N_{\mathrm{q}, \mathrm{det}}}
$$

where $N_{\mathrm{q}, \text { det }}$ is the number of blazars detected in a state of quiescence i.e. without any increase in flux of any blazar due to flaring.

In accordance with (Abdo et al. 2010c). with the parameter values corresponding to the best fit values for all blazars both FSRQs and BL LACs put together are $A_{F e r m i}=16.46 \times 10^{-14} \mathrm{~cm}^{2} \mathrm{sec} \mathrm{deg}{ }^{-2}, F_{\mathrm{b}}=6.60 \times 10^{-8} \mathrm{ph} \mathrm{cm}^{-2} \mathrm{sec}^{-1}$, $\beta_{1}=2.49$ and $\beta_{2}=1.58$. Picking a sensitivity higher than the break flux $F_{\text {sens }}=F_{\mathrm{b}} \eta_{\text {flare,eff }}$ as most certainly the blazars detectable are above the break, the value of $f \approx 5.7$ for a duty cycle, $\chi$ of $\sim 20 \%$ and a flaring factor of $\eta_{\text {flare }}=10$ assuming all flaring events have occurred. Instantaneously, however, the gain is simply $N_{\mathrm{u}} \times \chi$ which gives a factor of 2 for a uniform duty cycle of $20 \%$. However, as stated before the actual numbers would depend on a number of factors including the assumption that all blazars flare.

\section{REFERENCES}

Abdo, A. A., Ackermann, M., Ajello, M., et al. 2010a, Nature, 463, 919

-. 2010b, ApJ, 716, 835

—. 2010c, ApJ, 720, 435

Agudo, I., Thum, C., Wiesemeyer, H., \& Krichbaum, T. P. 2010, ApJS, 189, 1

Ajello, M., Alexander, D. M., Greiner, J., et al. 2012, ApJ, 749, 21

Angel, J. R. P., \& Stockman, H. S. 1980, ARA\&A, 18, 321

Angelakis, E., Fuhrmann, L., Nestoras, I., et al. 2010, ArXiv eprints

Baumgartner, W. H., Tueller, J., Markwardt, C. B., et al. 2013, ApJS, 207, 19

Bellazzini, R., Costa, E., Matt, G., \& Tagliaferri, G. 2010, X-ray Polarimetry

Bonometto, S., Cazzola, P., \& Saggion, A. 1970, A\&A, 7, 292

Chang, Z., Jiang, Y., \& Lin, H.-N. 2013, ApJ, 769, 70

Collmar, W., Böttcher, M., Krichbaum, T. P., et al. 2010, A\&A, 522, A 66

Costa, E., Bellazzini, R., Tagliaferri, G., et al. 2010, Experimental Astronomy, 28, 137

Dean, A. J., Clark, D. J., Stephen, J. B., et al. 2008, Science, 321, 1183

Fujiwara, M., Matsuoka, Y., \& Ienaka, N. 2012, AJ, 144, 112

Götz, D., Covino, S., Fernández-Soto, A., Laurent, P., \& Bošnjak, Ž. 2013, MNRAS, 431, 3550

Götz, D., Laurent, P., Lebrun, F., Daigne, F., \& Bošnjak, Ž. 2009, ApJ, 695, L208

Guo, Q., Beilicke, M., Garson, A., et al. 2013, Astroparticle Physics, 41, 63

Ikejiri, Y., Uemura, M., Sasada, M., et al. 2011, PASJ, 63, 639

Jorstad, S. G., Marscher, A. P., Larionov, V. M., et al. 2010, ApJ, 715,362

Jourdain, E., Götz, D., Westergaard, N. J., Natalucci, L., \& Roques, J. P. 2008, in Proceedings of the 7th INTEGRAL Workshop

Kalemci, E., Boggs, S., Wunderer, C., \& Jean, P. 2004, in ESA Special Publication, Vol. 552, 5th INTEGRAL Workshop on the INTEGRAL Universe, ed. V. Schoenfelder, G. Lichti, \& C. Winkler, 859

Kataoka, J., Madejski, G., Sikora, M., et al. 2008, ApJ, 672, 787
Kirsch, M. G., Briel, U. G., Burrows, D., et al. 2005, in Society of Photo-Optical Instrumentation Engineers (SPIE) Conference Series, Vol. 5898, UV, X-Ray, and Gamma-Ray Space Instrumentation for Astronomy XIV, ed. O. H. W. Siegmund, $22-33$

Krawczynski, H. 2012, ApJ, 744, 30

Krawczynski, H., Angelini, L., Baring, M., et al. 2013, ArXiv eprints

Krivonos, R., Revnivtsev, M., Tsygankov, S., et al. 2010a, A\&A, 519, A107

Krivonos, R., Tsygankov, S., Revnivtsev, M., et al. 2010b, A\&A, $523, \mathrm{~A} 61$

Lazzati, D. 2006, New Journal of Physics, 8, 131

Marscher, A. P., Jorstad, S. G., D'Arcangelo, F. D., et al. 2008, Nature, 452, 966

McConnell, M. L., Smith, D. M., Emslie, A. G., et al. 2003, in Bulletin of the American Astronomical Society, Vol. 35, AAS/Solar Physics Division Meeting \#34, 850

McGlynn, S., Clark, D. J., Dean, A. J., et al. 2007, A\&A, 466, 895 McNamara, A. L., Kuncic, Z., \& Wu, K. 2009, MNRAS, 395, 1507

Meyer, E. T., Fossati, G., Georganopoulos, M., \& Lister, M. L. 2012, ApJ, 752, L4

Mizuno, T. 2012, in COSPAR Meeting, Vol. 39, 39th COSPAR Scientific Assembly, 1259

Moran, P., Shearer, A., Gouiffes, C., \& Laurent, P. 2013, ArXiv e-prints

Nalewajko, K. 2013, MNRAS, 598

Netzer, H. 2013, The Physics and Evolution of Active Galactic Nuclei

Novick, R. 1975, Space Sci. Rev., 18, 389

Pearce, M., Florén, H.-G., Jackson, M., et al. 2012, ArXiv e-prints Pian, E., Foschini, L., Beckmann, V., et al. 2006, A\&A, 449, L21

Richards, J. L., Max-Moerbeck, W., Pavlidou, V., et al. 2011, ApJS, 194, 29

Rossi, E. M., Lazzati, D., Salmonson, J. D., \& Ghisellini, G. 2004, MNRAS, 354, 86

Rybicki, G. B., \& Lightman, A. P. 1986, Radiative Processes in Astrophysics

Sasada, M., Uemura, M., Fukazawa, Y., et al. 2011, PASJ, 63, 489

Strohmayer, T. E., \& Kallman, T. R. 2013, ApJ, 773, 103 
Ulrich, M.-H., Maraschi, L., \& Urry, C. M. 1997, ARA\&A, 35, 445

Vercellone, S., \& for the AGILE Team. 2012, ArXiv e-prints

Wardle, J. F. C., \& Kronberg, P. P. 1974, ApJ, 194, 249

Wehrle, A. E., Marscher, A. P., Jorstad, S. G., et al. 2012, ApJ, 758,72

Weisskopf, M. C., Elsner, R. F., \& O'Dell, S. L. 2010a, in Society of Photo-Optical Instrumentation Engineers (SPIE) Conference Series, Vol. 7732, Society of Photo-Optical Instrumentation Engineers (SPIE) Conference Series

Weisskopf, M. C., Guainazzi, M., Jahoda, K., et al. 2010b, ApJ, 713,912
Yonetoku, D., Murakami, T., Gunji, S., et al. 2011a, ApJ, 743, L30

Yonetoku, D., Murakami, T., Sakashita, T., et al. 2011b, in American Institute of Physics Conference Series, Vol. 1358, American Institute of Physics Conference Series, ed. J. E. McEnery, J. L. Racusin, \& N. Gehrels, 408-411 -. 2012, ApJ, 758, L1

Zacharias, M., \& Schlickeiser, R. 2012, MNRAS, 420, 84

Zhang, H., \& Böttcher, M. 2013, ApJ, 774, 18 